\title{
Unified Energy-Efficient Routing for Multi-hop Wireless Networks
}

\author{
Sungoh Kwon and Ness B. Shroff
}

\begin{abstract}
In this paper, we develop an energy-efficient routing scheme that takes into account three key wireless system elements: transmission power; interference; and residual energy. Since energy is a scarce resource, many energy-aware routing algorithms have been proposed to improve network performance. However, previous algorithms have been designed for a subset of these three main elements, which could limit their applicability. Thus, our contribution is here to develop a unified routing algorithm called the Energy-efficient Unified Routing (EURo) algorithm that accommodates any combination of these above key elements. We show via simulations that EURo outperforms the state-of-the-art.
\end{abstract}

Index Terms-multi-hop wireless network, energy-efficient routing, SINR, energy conservation, cross layer, simulations.

\section{INTRODUCTION}

Energy is a precious resource in wireless networks. For many multi-hop networking scenarios, nodes are batteryoperated, thus requiring efficient energy management to ensure connectivity across the network. Even when wireless networks are connected to power outlets, due to interference between active links the network may demand excessive energy per unit time (Power) so that the overall performance is reduced. Since energy efficiency is directly connected to the network life-time or network capacity, there have been many efforts to study energy-efficient networks in the wireless network community [1]. In the case of multi-hop wireless networks, such as wireless sensor networks [2] and wireless mesh networks [3], efficient routing algorithms are critical for network performance.

In previous works, three main metrics have been used for energy-efficient routing: transmission power, interference, and residual battery energy. However, previous works typically deal with either one or two of these metrics, as illustrated in Figure 1. For example, in [4], [5], energy-efficient routing mechanisms have been developed to find Minimum Energy (ME) routes in multi-hop wireless networks, but these algorithms do not account for the interference with other links nor battery energy. The authors in [6] study end-to-end QoS constraints, but do not consider the impact of routing a new flow

This work was done while both authors were at Purdue University, and has been supported in party by ARO MURI Award No. W911NF-07-10376 (SA08-03) and NSF grants ANI-0207728, 0626703-CNS, and 0635202-CCF.

S. Kwon is with Samsung Electronics Co., Dong Suwon P.O.BOX 105, 416 Maetan-3dong, Yeongtong-gu, Suwon-si, Gyeonggi-do, 443-742, Korea (email: sungoh@ieee.org)

N. B. Shroff is with the Departments of ECE and CSE, The Ohio State University, 2015 Neil Avenue, Columbus, OH 43210, U.S.A (email: shroff@ece.osu.edu)

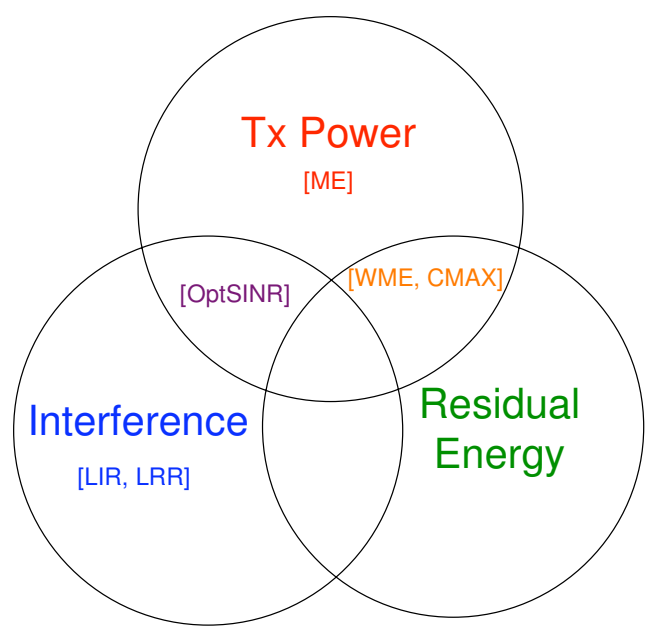

Fig. 1. Previous works for energy-efficient routing: ME, LIR, LRR, WME, CMAX, OptSINR.

on the interference and power requirements of the network, i.e., they do not consider how routing a new flow interferes with ongoing flows in the network. In [7], [8], [9], [10], the authors choose routes that use only interference between links as a metric for routing. In [7], [8] Least-Interference Routing (LIR) algorithms are developed to minimize the amount of interference caused by a transmission, while in [9], [10], Least-Resistance Routing (LRR) algorithms are developed to minimize the amount of interference encountered by a transmission. These algorithms in [7], [8], [9], [10] may result in choosing energy-inefficient routes because they do not explicitly consider energy efficiency and residual energy, but only interference. In [11], [12], [13], [14], the authors show that the residual energy plays an important role to improve network performance and propose the Weighted Minimum Energy (WME) routing algorithm [11] (it was called CMAX in [14]), but they do not consider interference among active links. In [15], the authors show that the interference between links significantly affects network performance and proposed the energy-efficient interference-based routing algorithm, called Optimal SINR Routing (OptSINR). However, the residual energy is not taken into account.

In practice, the three key elements of transmission power, interference, and residual battery energy play an important role in choosing energy-efficient routes. However, because previous studies have ignored one or more of these metrics, the resultant algorithms may not be energy efficient in a real wireless 
environment, where all of these elements play an important role. Thus, it is necessary to develop a simple and energyefficient algorithm that takes into account all of these critical metrics.

In this paper, we make the following contributions. We develop a unified energy-efficient routing algorithm that parameterizes all the three key metrics: transmission power, interference between links (or routes), and residual battery energy. We also show how the proposed algorithm works in different environments and how the proposed algorithm is related to other algorithms proposed in previous works. Throughout this paper, unless stated otherwise, we use boldface notation to denote either a matrix or a vector.

The rest of the paper is organized as follows. In Section II, we describe the system model and state our basic assumptions. In Section III, we develop an energy-efficient unified routing algorithm. In Section IV, we study the properties of the proposed routing algorithm in various environments. In Section $\mathrm{V}$, we provide numerical results to study the efficacy of the scheme. We conclude in Section VI.

\section{System Model AND Power Control}

\section{A. System model}

We consider a power-controlled wireless network that supports multi-hop routing, i.e., each node can control its transmission power. We further assume that flow dynamics are over a much larger time-scale than power control dynamics, so that the time required for power control to converge is negligible. The multi-hop wireless network is modeled as a directed graph $G=(\mathcal{N}, \mathcal{L})$, where $\mathcal{N}$ represents the set of nodes and $\mathcal{L}$ the set of edges that represent communication links between nodes in the network. Each node in $\mathcal{N}$ has initial energy $\varepsilon_{n}^{\text {init }}$ for $n \in \mathcal{N}$. Each link in $\mathcal{L}$ is identified by an ordered pair of nodes, i.e., the transmitting and receiving nodes. Links sharing the same frequency interfere with other links when simultaneously activated. A new service with data $\zeta$ to be transmitted requires a fixed data rate $\Delta q$, so that the service flow has a fixed duration $\mu$, i.e., $\mu=\frac{\zeta}{\Delta q}$. For this paper, we assume that a flow will be routed over only a single route for the entire duration of the flow. We define, $E(l)$, the energy consumption of link $l$ to be

$$
E(l)=P(l) \mu^{l},
$$

where $P(l)$ is the transmission power of link $l$ and $\mu^{l}$ is the amount of time it takes the flow to be served at link $l$. For simplicity, we do not consider energy replenishment considered in [11], [16]. However, we can readily modify the algorithm to include the energy replenishment case. The extension will be briefly discussed later.

\section{B. Wireless link model}

As mentioned earlier, due to the shared nature of the wireless medium, wireless links interfere with each other. The impact of interference affects the available capacity of these links.
We define a function $g(\cdot)$ that maps the achievable bandwidth (channel capacity) $r(l)$ to the corresponding signal-tointerference-and-noise-ratio (SINR) $\theta(l)$ as follows:

$$
\theta(l)=g(r(l))
$$

We assume that the function $g$ is increasing and differentiable with respect to $r(l)$, almost surely.

In the case of the band-limited additive white Gaussian noise (AWGN) channel, the channel capacity (also called the Shannon's capacity [17]) at link $l, r(l)$, is given by

$$
r(l)=B(l) \log (1+\theta(l)),
$$

where $B(l)$ represents the channel bandwidth at link $l$. Hence, (1) becomes

$$
\theta(l)=\exp \left(\frac{r(l)}{B(l)}\right)-1 .
$$

In the low SINR region, the available capacity in (1) is often assumed to be a linear function of SINR [18], that is expressed as

$$
\theta(l)=K r(l)
$$

where $K$ is a constant.

Since there exists a one-to-one mapping from a minimum bandwidth to the corresponding minimum SINR, as in (1), we replace a rate constraint by an SINR constraint as a measure of the minimum quality required on the link. We let $\Delta c$ denote an additional SINR constraint at a link when a new flow with an additional bandwidth $\Delta q$ comes into the link. In the case of the linear SINR regime, the additional SINR constraint $\Delta c$ is equal to $K \Delta q$ from (2).

The $\operatorname{SINR} \theta(l)$ at each link $l$ is defined as

$$
\begin{aligned}
\theta(l) & =\frac{G(T(l), R(l)) P(l)}{\sum_{m: m \neq l} P(m) G(T(m), R(l))+\sigma_{R(l)}} \\
& =\frac{G(T(l), R(l)) P(l)}{\eta_{R(l)}},
\end{aligned}
$$

where $T(l)$ is the transmitting node of link $l, R(l)$ is the receiving node corresponding to link $l, \sigma_{R(l)}$ is the ambient noise at node $R(l), P(l)$ is the transmission power at node $T(l)$, $G(T(m), R(l))$ is the path gain between transmitter $T(m)$ and receiver $R(l)$, and $\eta_{R(l)}$ is the sum of interference and noise at node $R(l)$. The path gain $G(T(m), R(l))$ is modeled as

$$
G(T(m), R(l))=K_{T(m) R(l)} d_{T(m) R(l)}^{-\delta},
$$

where $K_{T(m) R(l)}$ is the attenuation factor that models power loss due to shadowing, $d_{T(m) R(l)}$ is the distance between nodes $T(m)$ and $R(l)$, and $\delta$ is the path loss exponent that typically ranges between 2 and 6 [19].

\section{Power control}

Recall that $\mathcal{L}$ is the set of links. We let $\mathbf{P}$ denote the power vector defined by $\mathbf{P}=\left(P(1), \cdots, P\left(L_{\mathcal{L}}\right)\right)^{T}$, where $P(l)$ is the power of link $l$, and $L_{\mathcal{L}}$ is the number of links in set $\mathcal{L}$. 
Each link has a minimum requirement $c(l)$ in terms of SINR, i.e.,

$$
\theta(l) \geq c(l)
$$

Using (3), we can rewrite the above inequality (5) in matrix form as

$$
\mathbf{P} \geq \mathbf{F P}+\mathbf{b}
$$

where $\mathbf{b}=\left(b(1), \cdots, b\left(L_{\mathcal{L}}\right)\right)^{T}$ such that $b(l)=\frac{c(l) \sigma_{R(l)}}{G(T(l), R(l))}$, and $\mathbf{F}$ is the $L_{\mathcal{L}} \times L_{\mathcal{L}}$ matrix with $(l, m)$ entry

$$
F(l, m)= \begin{cases}\frac{G(T(m), R(l)) c(l)}{G(T(l), R(l))} & , l \neq m \\ 0 & , l=m .\end{cases}
$$

Matrix F defined by (7) has non-negative elements, and since the links interact with each other, it is also irreducible. Hence, we have the following theorem [20] from the PerronFrobenius theorem and standard matrix theory [21].

Theorem 1: The following statements are equivalent:

1) $\rho_{\mathbf{F}} \leq 1$ where $\rho_{\mathbf{F}}$ is the Perron-Frobenius eigenvalue of F.

2) There exists a vector $\mathbf{P}>0$ such that $(\mathbf{I}-\mathbf{F}) \mathbf{P} \geq \mathbf{b}$.

3) $(\mathbf{I}-\mathbf{F})^{-\mathbf{1}}$ exists and is positive componentwise.

If there exists a positive feasible vector $\mathbf{P}$, it follows from Theorem 1 that $(\mathbf{I}-\mathbf{F})^{-\mathbf{1}}$ exists. From (6) we obtain

$$
\mathbf{P} \geq(\mathbf{I}-\mathbf{F})^{-\mathbf{1}} \mathbf{b} \text {. }
$$

Hence, we have the Pareto optimal ${ }^{1}$ solution $(\mathbf{I}-\mathbf{F})^{-\mathbf{1}} \mathbf{b}$ that supports the network topology defined by links in $\mathcal{L}$, and their associated minimum requirements. One can use a distributed power control algorithm [20], [22] to achieve this minimum power vector. As is well known, link scheduling can improve network performance. However, in general, when a link scheduling algorithm is used, some links will not be activated. This means that those entries in $\mathbf{F}$, for which the links are not activated, will be zero so that $(\mathbf{I}-\mathbf{F})$ will not have a full rank. In this case, the optimal solution $(\mathbf{I}-\mathbf{F})^{-\mathbf{1}} \mathbf{b}$ can be found using a Moore-Penrose inverse matrix [23]. The entry of the solution has non-negative values when the link is activated, and zero otherwise.

We let $(\mathbf{I}-\mathbf{F})_{(l)}^{-1}$ denote the $l$ th column vector of matrix $(\mathbf{I}-\mathbf{F})^{-\mathbf{1}}$ and $(\mathbf{I}-\mathbf{F})_{\sum^{n}}^{-1}$ the element-wise sum of vector $(\mathbf{I}-\mathbf{F})_{(l)}^{-1}$. Then, the minimum energy increment $\Delta \mathbf{E}_{l}^{*}$ of each link in the network, when a new flow with additional constraint $\Delta c$ and duration $\mu$ arrives at link $l$, can be expressed as

$$
\begin{aligned}
\Delta \mathbf{E}_{l}^{*} & =\Delta \mathbf{P}_{l}^{*} \mu \\
& =\left(\mathbf{I}-\mathbf{F}^{\prime}\right)_{(l)}^{-1}\left(\frac{\eta_{R(l)}}{G(T(l), R(l))}\right) \Delta c \mu,
\end{aligned}
$$

where $\Delta \mathbf{P}_{l}^{*}$ is the minimum power addition required in the network to serve the new flow at link $l, \mathbf{F}^{\prime}$ is the matrix corresponding to $\mathbf{F}$ in the new environment, and $\eta_{R(l)}$ is the

\footnotetext{
${ }^{1} \mathbf{P}^{*}$ is said to be Pareto optimal if $\mathbf{P}^{*}$ is feasible and any feasible $\mathbf{P}$ satisfies $\mathbf{P} \geq \mathbf{P}^{*}$ componentwise.
}

sum of interference and noise at node $R(l)$. (See [24] for details of how to obtain (8))

Similarly, when a flow is served by a set $\Lambda$ of links at a given time slot, the increased energy consumption $\Delta \mathbf{E}_{\Lambda}$ can be expressed as

$$
\Delta \mathbf{E}_{\Lambda}^{*}=\sum_{l \in \Lambda}\left(\mathbf{I}-\mathbf{F}^{\prime}\right)_{(l)}^{-1}\left(\frac{\eta_{R(l)}}{G(T(l), R(l))}\right) \Delta c \mu .
$$

\section{Problem formulation}

The problem that we would like to solve is to find a route in order to maximize the throughput over some finite time period $[0, t]$, i.e., for a given scheduling policy $S$, we would like to solve the following problem:

$$
\begin{array}{rl}
(A) \quad \arg \max _{R} & J(P, S, R, \zeta, t) \\
\text { subject to } & \theta(l) \geq c(l) \quad \forall l \in \mathcal{L} \\
& P^{\max }(l) \geq P(l) \geq 0 \quad \forall l \in \mathcal{L}, \\
& \varepsilon_{n} \geq 0 \quad \forall n \in \mathcal{N},
\end{array}
$$

where $J(P, S, R, \zeta, t)$ is the number of successfully delivered packets over time duration $[0, t]$ when route $R$ is chosen as the new flow $\zeta, P$ is the transmission power, $\theta(l)$ is SINR at link $l, c(l)$ is the minimum requirement of link $l$ in terms of SINR, and $\varepsilon_{n}$ is the remaining energy at node $n$.

\section{UNIFIED ENERGY-EFFICIENT ROUTING (EURO) ALGORITHM}

To solve problem $(A)$, we use a two-step approach. We first review the case without interference constraints. We then add interference constraints to develop a unified routing algorithm. We begin without considering scheduling, and then extend the developed algorithm to the case when the links are randomly scheduled.

\section{A. Energy-efficient routing for battery-operated networks in the absence of interference}

In [11], it is shown that WME routing is an asymptotically optimal solution to problem $(A)$. In the presence of interference, WME can be expressed as follows.

$$
\begin{array}{ll}
\text { (B) } \arg \min _{R} & \sum_{l \in R} \mathbf{W} \Delta \mathbf{E}_{l} \\
\text { subject to } & P^{\max }(l) \geq P(l) \geq 0 \quad \forall l \in \mathcal{L}, \\
& \varepsilon_{n} \geq 0 \quad \forall n \in \mathcal{N},
\end{array}
$$

where $\mathbf{W}$ is a weight vector that is a function of the residual energy of nodes when a new flow arrives to the network, and $\Delta \mathbf{E}_{l}$ is the energy increment over the network when the new flow is served at link $l$. In the interference-free environment, the $l$ th entry of $\Delta \mathbf{E}_{l}$ is equal to the transmission energy increment of link $l$, which is used in [11], [14] when a new flow traverses over link $l$. The weight vector $\mathbf{W}$ is a row vector $\mathbf{W}=\left(W(1), W(2), \cdots, W\left(L_{\mathcal{L}}\right)\right)$. As in [11], [14], we define the $l$ th entry $W(l)$ as

$$
W(l)=\varepsilon_{T(l)}^{i n i t}\left(\gamma^{\lambda(l)}-1\right),
$$


where $\gamma$ is a constant, $\lambda(l)$ is the ratio of the depleted energy to the initial energy at the transmitter $T(l)$ of link $l$. In the case when energy replenishment is available (e.g. [11], [16]), the weight of (9) can be extended by adding a multiplication factor of the replenishment as shown in [11].

Let $\mathbf{E}^{*}$ be a Pareto optimal solution required to meet the SINR requirement. The weights defined in (9) are always nonnegative. Hence, for a given route $R$, we have the following inequality:

$$
\begin{aligned}
& \sum_{l \in R} \mathbf{W} \Delta \mathbf{E}_{l}-\sum_{l \in R} \mathbf{W} \Delta \mathbf{E}_{l}^{*} \\
= & \sum_{l \in R} \mathbf{W}\left(\Delta \mathbf{E}_{l}-\Delta \mathbf{E}_{l}^{*}\right) \\
= & \sum_{l \in R} \mathbf{W}\left(\left(\mathbf{E}_{l}-\mathbf{E}\right)-\left(\mathbf{E}_{l}^{*}-\mathbf{E}\right)\right) \\
= & \sum_{l \in R} \mathbf{W}\left(\mathbf{E}_{l}-\mathbf{E}_{l}^{*}\right) \\
\geq & 0,
\end{aligned}
$$

where $\mathbf{E}, \mathbf{E}_{l}$, and $\mathbf{E}_{l}^{*}$ represent the present transmission power of links, the transmission power of links when a new flow comes into link $l$, and the Pareto optimal transmission power of links when a new flow comes into link $l$, respectively. Hence, the Pareto optimal $\mathbf{E}^{*}$ makes the weighted energy also Pareto optimal.

\section{B. Unified energy-efficient routing}

We now are in a position to solve problem (A), which is really the problem $(\mathrm{B})$ we considered above, but with interference constraints. Since the Pareto optimal energy makes the weighted energy Pareto optimal, as explained in the previous subsection, our energy-efficient solution to $(A)$ is expressed as

$$
\begin{aligned}
& \text { (C) } \quad \arg \min _{R} \sum_{l \in R} \mathbf{W} \Delta \mathbf{E}_{l}^{*} \\
& \text { subject to } P^{\max }(l) \geq P(l) \geq 0 \quad \forall l \in \mathcal{L} \text {, } \\
& \theta(l) \geq c(l) \quad \forall l \in \mathcal{L}, \\
& \varepsilon_{n} \geq 0 \quad \forall n \in \mathcal{N},
\end{aligned}
$$

where $\Delta \mathbf{E}_{l}^{*}$ stands for the minimum energy increase over the network for the new incoming flows at link $l$. As $\Delta c$ goes to zero, $\mathbf{F}^{\prime}$ in (8) converges to $\mathbf{F}$ element-wise so that the minimum energy $\Delta \mathbf{E}_{l}^{*}$ that meets all the SINR constraints becomes

$$
\Delta \mathbf{E}_{l}^{*}=(\mathbf{I}-\mathbf{F})_{(l)}^{-1}\left(\frac{\eta_{R(l)}}{G(T(l), R(l))}\right) \Delta c \mu .
$$

When the minimum SINR for the incoming flow is infinitesimally small, our energy-efficient routing algorithm can be formally expressed as

$$
\begin{aligned}
\arg \min _{R \in R(i, j)} & \sum_{l \in R}\left(\mathbf{W}(\mathbf{I}-\mathbf{F})_{(l)}^{-1}\left(\frac{\eta_{R(l)}}{G(T(l), R(l))}\right)\right)(11) \\
\text { subject to } & P^{\max }(l) \geq P(l) \geq 0 \quad \forall l \in \mathcal{L}, \\
& \theta(l) \geq c(l) \quad \forall l \in \mathcal{L}, \\
& \varepsilon_{n} \geq 0 \quad \forall n \in \mathcal{N},
\end{aligned}
$$

where $R(i, j)$ is the set of possible routes from node $i$ to node $j$. Instead of $\Delta \mathbf{E}_{l}$, the exact value of the network energy increment over a route, we can choose a minimum energy route from the interference measured at $R(l), \frac{\eta_{R(l)}}{G(T(l), R(l))}$, and $(\mathbf{I}-\mathbf{F})_{(l)}^{-1}$, as described below.

\section{Energy-efficient Unified Routing (EURo) algorithm}

Construct a directed graph $G=(\mathcal{N}, \mathcal{L})$.

For an incoming flow, check if resources are available.

If yes,

Measure the interference strength at all nodes in $\mathcal{N}$.

Calculate $(\mathbf{I}-\mathbf{F})^{-\mathbf{1}}$ based on path loss and constraints.

Calculate the present weight vector $\mathbf{W}$ as in (9).

Calculate link cost $\mathbf{W}(\mathbf{I}-\mathbf{F})_{(l)}^{-1}\left(\frac{\eta_{R(l)}}{G(T(l), R(l))}\right) \forall l \in \mathcal{L}$.

Apply a shortest path algorithm, e.g. Dijkstra's algorithm or

Bellman-Ford algorithm, to find the minimum cost route. Otherwise,

Reject the incoming flow.

Notify the rejection to the source.

In the EURo algorithm outlined above, each node checks the availability of two resources for an incoming flow: battery energy and transmission power. If the better energy is depleted or the transmission power is saturated at a node, the node denies the incoming flow.

We now assume that links are randomly scheduled and that the statistics of the links are available at each node. Let $\Pi$ be an $L_{\mathcal{L}} \times L_{\mathcal{L}}$ diagonal matrix such that the $(l, l)$ entry of $\Pi$ is defined as the probability that link $l$ is activated. We let $\pi_{m \mid l}$ denote the conditional probability that link $m$ is active given that link $l$ is active. From [15], the additional expected network energy $\Delta \overline{\mathbf{E}}_{l}$ is given by

$$
\Delta \overline{\mathbf{E}}_{l}^{*}=\left(\boldsymbol{\Pi}(\mathbf{I}-\overline{\mathbf{F}})^{-\mathbf{1}}\right)_{(l)}\left(\frac{\bar{\eta}_{R(l)}}{G(T(l), R(l))}\right) \Delta c \mu,
$$

where $\bar{\eta}_{R(n)}$ is the average of the interference and noise measured at the receiving node of link $n$ when link $n$ is active, and $\overline{\mathbf{F}}$ is an $L_{\mathcal{L}} \times L_{\mathcal{L}}$ matrix with entry $(l, m)$ defined as

$$
F(l, m)= \begin{cases}\frac{\pi_{m \mid l} G(T(m), R(l)) c(l)}{G(T(l), R(l))} & , l \neq m \\ 0 & , l=m\end{cases}
$$

Hence, our energy-efficient routing algorithm for random link scheduling schemes can be expressed as

$$
\begin{aligned}
\arg \min _{R \in R(i, j)} & \sum_{l \in R}\left(\mathbf{W}\left(\boldsymbol{\Pi}(\mathbf{I}-\overline{\mathbf{F}})^{-1}\right)_{(l)} \frac{\bar{\eta}_{R(l)}}{G(T(l), R(l))}\right) \\
\text { subject to } & P^{\max }(l) \geq P(l) \geq 0 \quad \forall l \in \mathcal{L}, \\
& \theta(l) \geq c(l) \quad \forall l \in \mathcal{L}, \\
& \varepsilon_{n} \geq 0 \quad \forall n \in \mathcal{N} .
\end{aligned}
$$

The algorithm procedure is also similar to the procedure that we have discussed above. 


\section{Distributed algorithm}

Our routing algorithm (11) described in the previous subsection requires global information such as $\mathbf{W}$ and $(\mathbf{I}-\mathbf{F})_{(l)}^{-1}$. The global information for link costs does not lead itself immediately to distributed implementation. The computational complexity for link costs exponentially increase as the number of nodes goes by. However, since wireless signal strength exponentially decays in terms of distance, as in (4), distant wireless links barely affect each other. In large networks, update information could be also stale. Hence, gathering global information is not effective.

To reduce the computational complexity, we define the information range as the range for a node to locally disseminate its information (e.g. residual energy and minimum SINR to transmit) to neighboring nodes in the range, as depicted in Figure 2. The computational complexity of the local information is independent of the network size but depends on only the number of neighboring nodes to share information.

For a distributed version of EURo, we can employ a distributed shortest path routing algorithm such as the BellmanFord algorithm [25]. For link costs in (11), we use $\tilde{\mathbf{W}}$ and $(\mathbf{I}-\tilde{\mathbf{F}})^{-1}$, instead of $\mathbf{W}$ and $(\mathbf{I}-\mathbf{F})^{-1}$ that need global information, where $\tilde{\mathbf{W}}$ and $(\mathbf{I}-\tilde{\mathbf{F}})^{-1}$ are correspondingly reduced matrices using local information from nodes in the preset information range of a node. The interference and noise strength $\eta_{R(l)}$ and the path gain $G(T(l), R(l))$ in (11) are locally measurable [19]. Hence, each node can locally compute link costs for the distributed algorithm.

Nodes in the network can update the information for routing in several ways [26]. A simple method is that nodes periodically broadcast their status to other nodes, and their neighboring nodes update their stored information. Another way is to use piggy-backing. When the status information is relatively small compared to the data being transmitted, each node attaches its information to transmitting data in order to disseminate the information. A control channel can be used for information distribution. The combination of these methods is also an alternative option to implement.

\section{Properties of the Unified Routing Algorithm}

We study the properties of the unified routing algorithm proposed in the previous section.

The metric used in our routing algorithm (11) is composed of the product of three components that represent residual energy, transmission power, and the impact of the transmission power. In various wireless environments as summarized in Table I, we study how each component plays a role and show how our routing algorithm relates to other previous routing algorithms.

\section{A. No interference and infinite energy}

We assume here that interference is negligible and that each node's energy is unlimited. For example, a system is connected to an outlet and the arrival rate into the system is very low.

Since there is no interference, matrix $\mathbf{F}$ becomes a zero matrix. The unlimited energy constraint means that the weight
$W(l)$ is constant for all links. In the case of homogeneous networks, the ambient noise is identical. Hence the algorithm becomes

$$
\begin{aligned}
& \arg \min _{R \in R(i, j)} \sum_{l \in R}\left(\mathbf{W}(\mathbf{I}-\mathbf{F})_{(l)}^{-1}\left(\frac{\eta_{R(l)}}{G(T(l), R(l))}\right)\right) \\
= & \arg \min _{R \in R(i, j)} \sum_{l \in R}\left(\frac{\sigma_{R(l)}}{G(T(l), R(l))}\right) \\
= & \arg \min _{R \in R(i, j)} \sum_{l \in R}\left(\frac{1}{G(T(l), R(l))}\right) .
\end{aligned}
$$

This is then the same as the Minimum Energy routing algorithm so that under this assumption our routing algorithm performs at least as well as the Minimum Energy routing algorithm.

\section{B. No interference with energy limitation}

We assume that interference is negligible and that each node is operated using a battery. In this case the weight of each node depends on the residual energy. However, since there is no interference, matrix $\mathbf{F}$ becomes zero as in the previous case. Hence, in this environment our routing algorithm can be simplified as

$$
\begin{aligned}
& \arg \min _{R \in R(i, j)} \sum_{l \in R}\left(\mathbf{W}(\mathbf{I}-\mathbf{F})_{(l)}^{-1}\left(\frac{\eta_{R(l)}}{G(T(l), R(l))}\right)\right) \\
= & \arg \min _{R \in R(i, j)} \sum_{l \in R}\left(W(l) \frac{\sigma_{R(l)}}{G(T(l), R(l))}\right) .
\end{aligned}
$$

This algorithm is now identical to the Weighted Minimum Energy routing algorithm, given in [11], [14]. In this wireless environment our routing algorithm can distribute relay load over the network in terms of residual energy so that the performance of our routing algorithm equals that of the Weighted Minimum Energy routing algorithm.

\section{Interference and infinite energy}

We assume that interference is significant and that each node is connected to a power outlet such as a wireless mesh network with a significant incoming flow rate.

Since there is no constraint on the available energy (power outlet is available), the weight of each node in (11) is identical. Hence our routing algorithm can now be expressed as

$$
\begin{aligned}
& \arg \min _{R \in R(i, j)} \sum_{l \in R}\left(\mathbf{W}(\mathbf{I}-\mathbf{F})_{(l)}^{-1}\left(\frac{\eta_{R(l)}}{G(T(l), R(l))}\right)\right) \\
= & \arg \min _{R \in R(i, j)} \sum_{l \in R}\left((\mathbf{I}-\mathbf{F})_{\sum^{l}}^{-1}\left(\frac{\eta_{R(l)}}{G(T(l), R(l))}\right)\right),
\end{aligned}
$$

which is identical to the OptSINR routing algorithm [15]. In this wireless environment, our routing algorithm performs as well as OptSINR. 
TABLE I

COMPARISON OF ALGORITHMS: WE CONSIDER FIVE DIFFERENT ENVIRONMENTS DEPENDING ON CONSIDERED METRIC. WE MARK WITH AN O WHEN AN ALGORITHM CONSIDERS THAT METRIC, OTHERWISE, WE MARK AN X.

\begin{tabular}{|c|c|c|c|c|}
\hline \multirow{2}{*}{ Case (Section) } & \multicolumn{3}{|c|}{ Measure elements } & \multirow{2}{*}{ Algorithms } \\
\cline { 2 - 4 } & Interference & Transmission power & Residual energy & \\
\hline 1 (IV-A) & $\mathrm{X}$ & $\mathrm{O}$ & $\mathrm{X}$ & ME \\
\hline 2 (IV-B) & $\mathrm{X}$ & $\mathrm{O}$ & $\mathrm{O}$ & WME, CMAX \\
\hline 3 (IV-C) & $\mathrm{O}$ & $\mathrm{O}$ & $\mathrm{X}$ & OptSINR \\
\hline 4 (IV-D) & $\mathrm{O}$ & $\mathrm{X}$ & $\mathrm{X}$ & LIR, LRR \\
\hline 5 & $\mathrm{O}$ & $\mathrm{O}$ & $\mathrm{O}$ & EURo \\
\hline
\end{tabular}

\section{Interference only}

We consider the case when only interference is used as the metric for choosing a route. The case can be categorized into two cases: minimize the interference experienced by a route, and minimize the interference induced by a route.

To study routing algorithms that consider only interference, we need to investigate the physical meaning of (10). Since $(\mathbf{I}-\mathbf{F})^{-\mathbf{1}}=\mathbf{I}+\mathbf{F}+\mathbf{F}^{\mathbf{2}}+\mathbf{F}^{\mathbf{3}}+\cdots$, the additional energy over the network can be rewritten as follows.

$$
\begin{aligned}
& \Delta \mathbf{E}_{l}^{*} \\
= & \left(\mathbf{I}_{\sum^{l}}+\mathbf{F}_{\sum^{l}}+\mathbf{F}_{\sum^{l}}^{2}+\cdots\right)\left(\frac{\eta_{R(l)}}{G(T(l), R(l))}\right) \Delta c \mu \\
= & (\underbrace{0, \cdots, 0}_{l-1},\left(\frac{\eta_{R(l)}}{G(T(l), R(l))}\right) \Delta c \mu, \underbrace{0, \cdots, 0}_{L_{\mathcal{L}}-l})^{T} \\
& +\mathbf{F}_{\sum^{l}}\left(\frac{\eta_{R(l)}}{G(T(l), R(l))}\right) \Delta c \mu \\
& +\mathbf{F}_{\sum^{l}}^{2}\left(\frac{\eta_{R(l)}}{G(T(l), R(l))}\right) \Delta c \mu+\cdots .
\end{aligned}
$$

The first term in (12) represents the amount of additional energy at each link when link $l$ serves a new flow with additional constraint $\Delta c$. The second term is the amount of additional energy over the network when link $l$ increases its transmission energy by the first term to serve the new incoming flow. In the same way, each term in (12) represents the iterated energy over the network induced by interference.

The routing algorithm to minimize the interference experienced by a route, called LRR [9], can be expressed as

$$
\arg \min _{R \in R(i, j)} \sum_{l \in R} \eta_{R(l)},
$$

where $\eta_{R(l)}$ is the interference measured at the receiver node $R(l)$ of link $l$.

From Equation (12), the routing algorithm minimizing the experienced interference is the same as the minimization of the element-wise summation of only the first terms when the path gain of link $l$ is ignored. Since the impact of the interference on the network (the second and higher order terms in (12)) and the path gain are ignored, the routing algorithm could choose less energy-efficient routes than OptSINR and our unified energyefficient routing algorithm.

In [7], [8], the following routing algorithm is used to

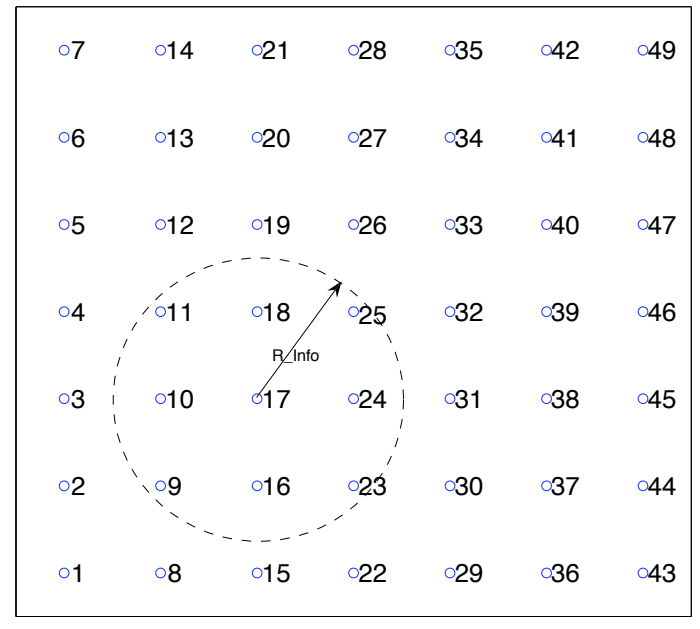

Fig. 2. System setting: a seven by seven grid network. R_Info represents the radius of a information range for a distributed algorithm

minimize the interference induced by a route, called $\mathrm{LIR}^{2}$ in [7],

$$
\arg \min _{R \in R(i, j)} \sum_{l \in R}\left(\sum_{m \in \mathcal{L}-\{l\}} G(T(l), R(m))\right),
$$

where $G(T(l), R(m))$ is the path gain from the transmitter of link $l$ to the receiver of link $m$. This algorithm (13) corresponds to (12), when we fix $G(T(l), R(l)) \forall l \in \mathcal{L}$ to be a constant value and ignore the transmission power of link $l$ (the first term in (12)) and the impact of interference on the network (the third and higher order terms in (12)). Thus, the routing algorithm (13) results in choosing less energy-efficient routes than OptSINR as well as our routing algorithm.

From IV-A, IV-B, IV-C, and IV-D, we show that our routing algorithm includes all the factors considered in the previous works and adapts to different network environments. Furthermore, our routing algorithm performs better than or at least as well as the other algorithms that are designed under marginal environments.

\footnotetext{
${ }^{2}$ The original routing algorithm proposed in [7] defines the potential interference as the number of links of which interference levels are above a certain threshold. To generalize the problem, we use here the total amount of interference level.
} 


\section{Simulations}

In this section we use simulations to verify the performance of our algorithm EURo. We compare the performance of EURo to WME, LIR, and ME. Other algorithms have been extensively studied in comparison with WME and OptSINR in [11], [15], and WME and OptSINR have been found to perform better than these other algorithms. Therefore, in this section we compare the performance only between these four algorithms. In the first three scenarios, we consider the four algorithms with global information to obtain the achievable performance in each marginal environment. In the final scenario, we compare distributed EURo (dEURo) with other four algorithms (EURo, WME, LIR, and ME).

For all the algorithms compared, we assume that the nodes employ power control. In each case, when sending flows through the routes chosen by the algorithm, each node adjusts its transmission power to satisfy the new minimum constraint at the links. For the simulations, we use a seven by seven grid network, and the separation between adjacent nodes in the $x$ - and $y$ - coordinates is one unit of distance, as depicted in Figure 2. We fix the path loss exponent at three, the attenuation factor at one, and the ambient noise at one. We assume that all ambient noise is identical and that each link is directional. We assume that wireless links are linear, as defined in (2), and that the required SINR of a new service flow is fixed at 0.1($10 \mathrm{~dB}$ ). In an IS-95 direct sequence code division multiple access (DS-CDMA) system with bandwidth $1.23 \mathrm{MHz}$, the minimum SINR for a $9.6 \mathrm{kbps}$ channel is $-14 \mathrm{~dB}(0.3981)$ [27]. We set a minimum SINR for each incoming service flow at $-10 \mathrm{~dB}$ in this simulation.

For link scheduling, we use the fixed and periodic link scheduling scheme used in [15], [28]. We fix $\gamma$ in (9) at 200 and a packet length at 200 slots. For each simulation, we use 10 different random seeds and average the performance.

For dEURo, we fix the information range of each node at 1.5 distance units, as illustrated in Figure 2, so that only adjacent neighboring nodes can share the information to compute link costs for distributed shortest path routing. To update local information, each node periodically announces its status information to its neighbors via a control channel.

We define the number of partitions as the number of transmission failures due to depleted relay nodes and use this measure to compare the performance of the various schemes, as in [11].

\section{A. No interference}

We first consider the case when there is no interference between routes. The service times of flows here do not overlap in the networks so that routes do not interfere with each other, and each node initially has 10 units of energy. In this case, the metrics of WME and EURo are identical so that EURo works the same as WME, as shown in Figures 3 and 4. Because they take into account residual energy in their cost functions, WME and EURo outperform LIR and ME. Since LIR can distribute load over the network better than ME, for a given number

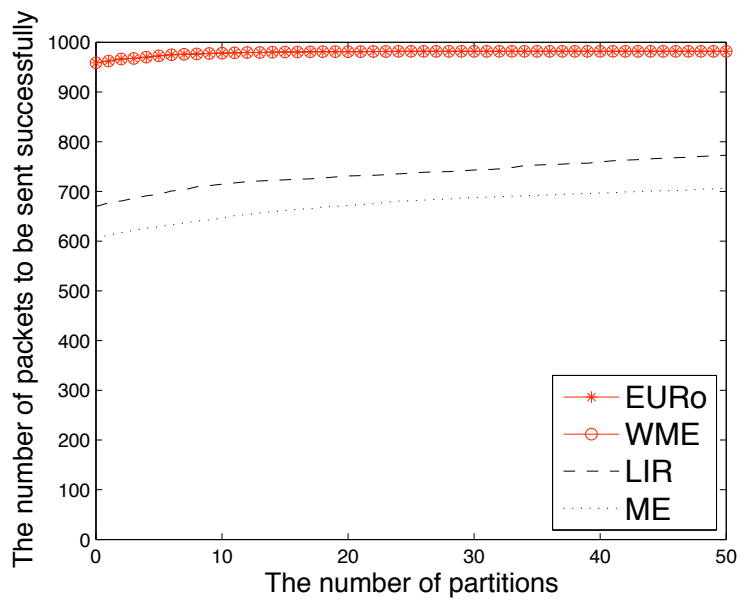

Fig. 3. Accumulated throughput versus the number of partitions when there exists no interference between links

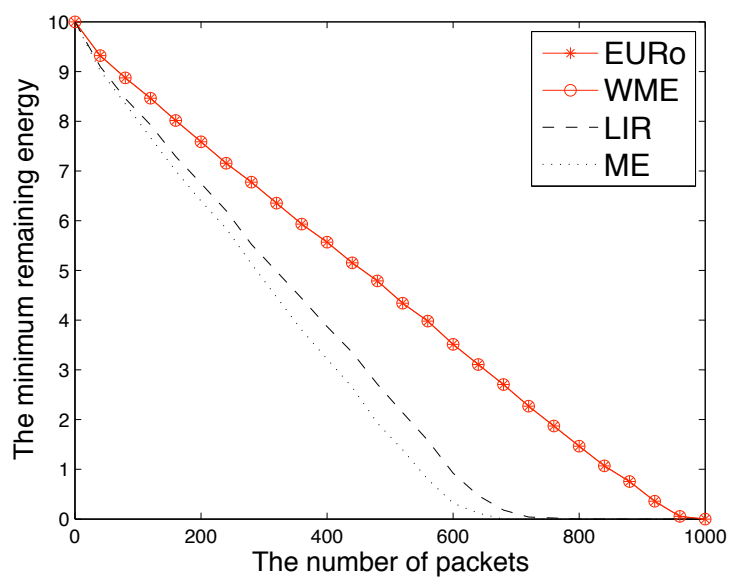

Fig. 4. The minimum remaining energy versus the number of arrived packets when there exists no interference between links

of node partitions, LIR successfully delivers more packets to their destinations than ME.

\section{B. Impact of interference}

In this subsection, we consider the impact of interference on the algorithms. We assume that the initial battery energy of each node is 10 units of energy. For simplicity, we fix the ongoing link from node 39 to node 46 in Figure 2. The ongoing link continuously transmits a flow with $2 \mathrm{~dB}$ SINR. The arrival rate is assumed to be small enough for flows to not overlap. In this environment, EURo outperforms the other algorithms.

Figure 5 shows the number of successfully delivered flows versus the number of partitions. EURo is almost constant over the number of partitions. However, due to interference, routes chosen by WME expend more energy than those of EURo, so that WME results in a smaller throughput. Compared to the previous case V-A, WME still performs better than LIR, but the performance differential is reduced. 


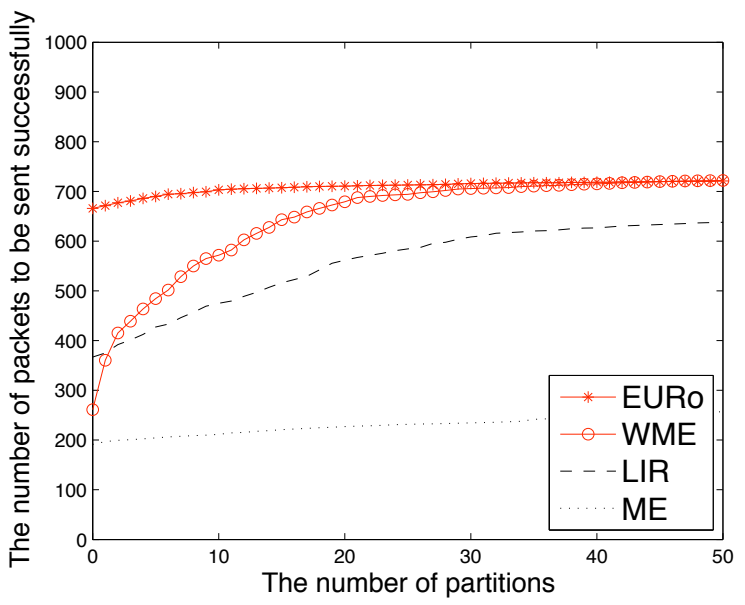

Fig. 5. Accumulated throughput versus the number of partitions when links interfere with each other

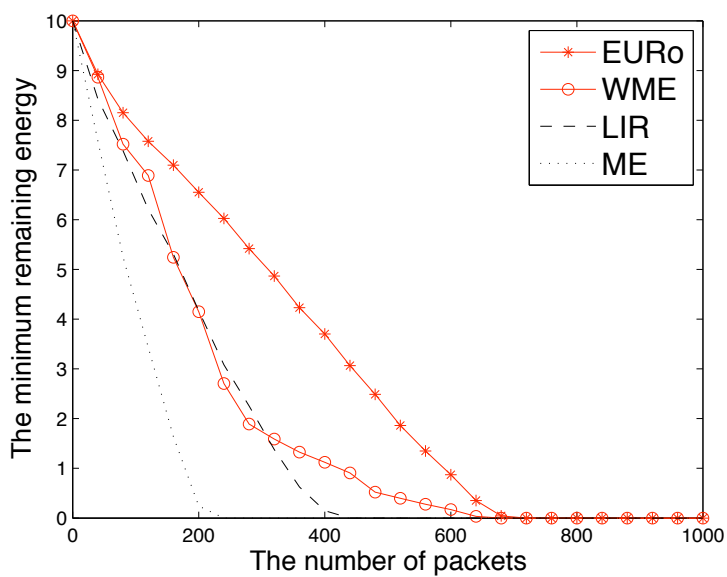

Fig. 6. The minimum remaining energy versus the number of arrived packets when links interfere with each other

Figure 6 shows the minimum energy among the nodes in the network after every transmission. Since the impact of interference between the ongoing link and new routes, the energy consumption rate of WME is steeper than EURo, which considers the residual energy and the interference.

\section{Impact of unevenly distributed initial energy}

This scenario includes heterogeneous sensor networks. Even in homogenous network environments, multiple deployments of nodes can make the initial battery levels uneven. Under the same environment as in the previous subsection, we consider the impact of unevenly distributed initial battery energy. We assume that the initial battery energy of the network follows a uniform distribution between 5 and 15 units of energy so that the mean of the distribution is 10 units of energy. Due to the variation of battery energy, the weights $W(l)$ in the algorithm play a more important role for choosing a route than those in the homogeneous battery case. Hence the performance of WME shown in Figures 7 and 8 is closer to the performance

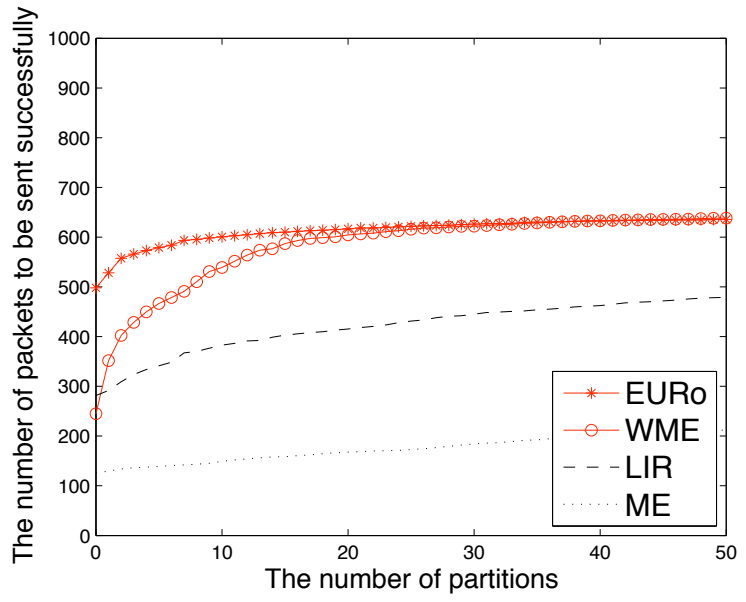

Fig. 7. Accumulated throughput versus the number of partitions when battery energy is unevenly distributed over the network

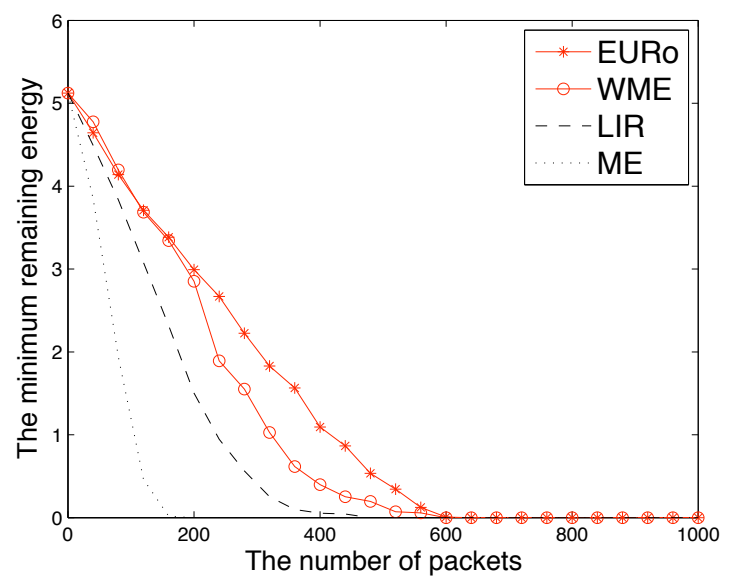

Fig. 8. The minimum remaining energy versus the number of arrived packets when battery energy is unevenly distributed over the network

of EURo compared to the previous case V-B.

\section{Impact of random arrival flows}

To study the impact of random flow arrivals on the routing algorithms, we fix the initial energy of each node at 10 units and compare the performance with two different arrival rates of 0.025 and 0.5 packet per slot, as shown Figures 9 and 10 . When the arrival rate is low, the average of the flows in the network is low so that the performance is close to, but slightly poorer than the performance in the case when there is no interference, as in Figure 9. In the case when the arrival rate is high, due to interference between the links, the algorithms that do not consider the impact of interference are more affected than EURo, as shown in Figure 10. Even if EURo uses only local information from adjacent neighborhoods, it outperforms other routing algorithms. As we would expect, the performance of dEURo the distributed version which uses only truncated information, is slightly poorer than EURo. 


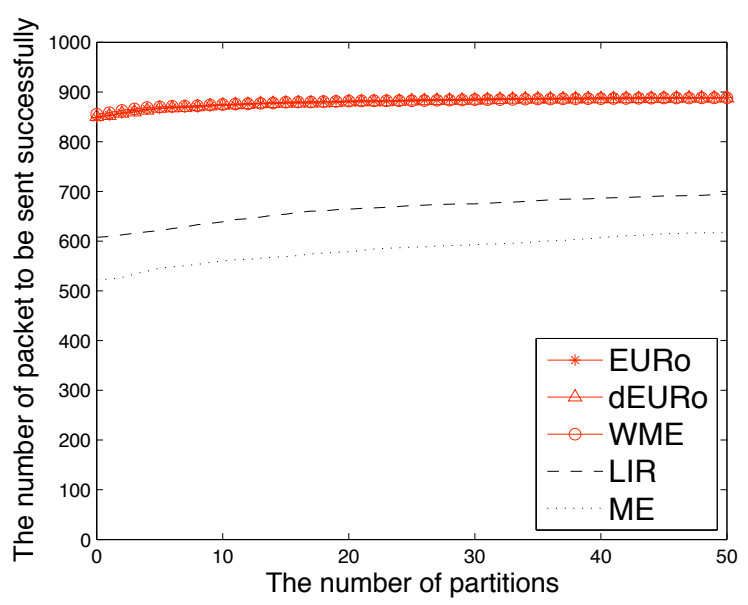

Fig. 9. Accumulated throughput versus the number of partitions when links interfere with each other when arrival rate is 0.025 packet per slot

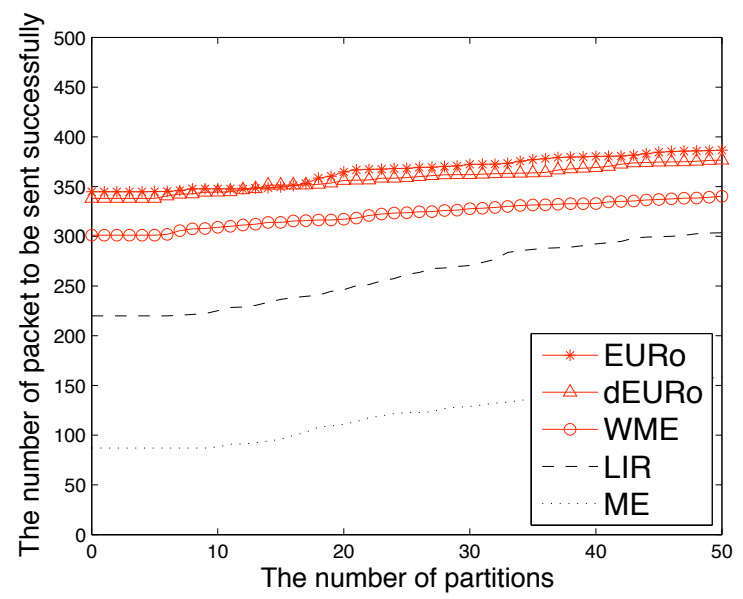

Fig. 10. Accumulated throughput versus the number of partitions when links interfere with each other when arrival rate is 0.5 packet per slot

\section{CONCLUSiON}

In this paper, we have developed EURo, an energy-efficient unified routing scheme. Unlike previous works, the proposed algorithm simultaneously takes into account three critical system parameters: transmission power, interference, and residual energy. We show that our algorithm maps to the state of the art, when certain quantities are kept fixed. Via simulations we show that our algorithm outperforms other energy-efficient routing algorithm in various environments. Even distributed version of EURo that uses local information outperforms other routing algorithms that use only a subset of the three system metrics.

\section{REFERENCES}

[1] C. E. Jones, K. M. Sivalingam, and P. Agrawal, "A survey of energy efficient network protocols for wireless networks," ACM Journal of Wireless Networks (WINET), vol. 7, no. 4, July 2001.

[2] I. F. Akyildiz, W. Sue, Y. Sankarasubarmaniam, and E. Cayirci, "A survey on sensor networks," IEEE Communications Magazine, vol. 40, no. 8, pp. 102-114, August 2002.
[3] I. F. Akyildiz, X. Wang, and W. Wang, "Wireless mesh networks: a survey," Computer Networks, vol. 47, no. 4, pp. 445-487, March 2005.

[4] T. Hou and V. O. K. Li, "Transmission range control in multihop packet radio networks," IEEE Transactions on Communications, vol. 34, no. 1, pp. 38-44, 1986.

[5] T. Melodia, D. Pompili, and I. F. Akyildiz, "Optimal local topology knowledge for energy efficient geographical routing in sensor networks," in IEEE INFOCOM'04, March 2004, vol. 3, pp. 1705-1716.

[6] R. Manohar and A. Scaglione, "Power optimal routing in wireless networks," in IEEE International Conference on Communications (ICC)'03, May 2003, vol. 4, pp. 2979-2984.

[7] J. Stevens, Spatial Reuse through Dynamic Power and Routing Control in Common- Channel Random-Access Packet Radio Networks, Ph.D. thesis, University of Texas at Dallas, 1988.

[8] H. Wei, S. Ganguly, R. Izmailov, and Z. J. Hass, "Interference-aware ieee 802.16 wimax mesh networks," in IEEE VTC'05-Spring, May-June 2005, vol. 5, pp. $3102-3106$.

[9] M. B. Pursley and H. B. Russell, "Routing in frequency-hop packet radio networks with partial-band jamming," IEEE Transactions on Communications, vol. 41, no. 7, pp. 1117-1124, 1993.

[10] J. Tang, G. Xue, C. Chandler, and W. Zhang, "Interference-aware routing in multihop wireless networks using directional antennas," in IEEE INFOCOM'05, March 2005, vol. 1, pp. 751-260.

[11] L. Lin, N. B. Shroff, and R. Srikant, "Asymptotically optimal poweraware routing for multihop wireless networks with renewable energy sources," in IEEE INFOCOM'05, March 2005, vol. 2, pp. 1262-1272.

[12] J-H. Chang and L. Tassiulas, "Energy conserving routing in wireless ad-hoc networks," in IEEE INFOCOM'O0, May 2000, vol. 1, pp. $22-$ 31.

[13] Q. Li, J. Aslam, and D. Rus, "On-line power-aware routing in wireless ad-hoc networks," in ACM MobiCom'01, July 2001, pp. 108-121.

[14] K. Kar, M. Kodialam, T. V. Lakshman, and L. Tassiulas, "Routing for network capacity maximization in energy-constrained ad-hoc networks," in IEEE INFOCOM'03, April 2003, vol. 1, pp. 673-681.

[15] S. Kwon and N. B. Shroff, "Energy efficient interference-based routing for multi-hop wireless networks," in IEEE INFOCOM'06, April 2006, vol. 3, pp. 1350-1361.

[16] A. Kansal and M. B. Srivastava, "An environmental energy harvesting framework for sensor networks," in the 2003 international symposium on Low power electronics and design (ISLPED 2003), August 2003, pp. $481-486$.

[17] J. G. Proakis, Digital Communications, McGraw-Hill, New York, 3rd edition, 1995

[18] X. Lin and N. B. Shroff, "The impact of imperfect scheduling on cross-layer rate control in multihop wireless networks," in IEEE INFOCOM'05, March 2005, vol. 3, pp. 1804-1814.

[19] T. S. Rappaport, Wireless Communications: Principles and Practice, Prantice-Hall, Inc., New Jersey, 1996.

[20] N. D. Bambos, S. C. Chen, and G. J. Pottie, "Radio link admission algorithm for wireless networks with power control and active link quality protection," in IEEE INFOCOM'95, April 1995, vol. 1, pp. $97-104$.

[21] E. Seneta, Nonnegative Matrices and Markov Chains, Springer, New York, 1981.

[22] G. J. Foschini and Z. Miljanic, "A simple distributed autonomous power control algorithm and its convergence," IEEE Transactions on Vehicular Technology, vol. 42, no. 4, pp. 641-646, November 1993.

[23] E. K. P. Chong and S. H. Zak, An Introduction to Optimization, John Wiley and Sons, Inc., New York, 2nd edition, 2001.

[24] S. Kwon and N. B. Shroff, "Unified energy-efficient routing for multihop wireless networks," Technical Report, Purdue University, 2007, Available at http://min.ecn.purdue.edu/ sungoh/.

[25] D. Bertsekas and R. Gallager, Data Networks, Prentice-Hall Inc., New Jersey, 2nd edition, 1992.

[26] B. Karp, Geographic Routing for Wireless Networks, Ph.D. thesis, Harvard University, 2000

[27] W. C. Y. Lee, Mobile Cellular Telecommunications: Analog and digital systems, McGraw-Hill, New York, 2nd edition, 1995.

[28] F. Baccelli, N. Bambos, and C. Chan, "Optimal power, throughput and routing for wireless link arrays," in IEEE INFOCOM'06, April 2006, vol. 3, pp. 1374-1385. 